\title{
New phenomenon of neutrino spin oscillations in transversal matter currents
}

\author{
Alexander Studenikin* \\ Department of Theoretical Physics, Faculty of Physics, Lomonosov Moscow State University, \\ Moscow 119991, Russia \\ Joint Institute for Nuclear Research, Dubna 141980, Moscow Region, Russia \\ E-mail: studenikdsrd.sinp.msu.ru
}

\begin{abstract}
After a brief history of neutrino mixing and oscillations, including neutrino spin oscillations, we develop quantum treatment of new phenomenon of neutrino spin oscillations engendered by the transversal matter currents. Possibilities for the enhancement of the effect by the longitudinal matter currents and longitudinal magnetic fields are analyzed.
\end{abstract}

Corfu Summer Institute 2017 "School and Workshops on Elementary Particle Physics and Gravity", 2 - 28 September, 2017,

Corfu, Greece

\footnotetext{
* Speaker.
} 


\section{Introduction: a brief history of neutrino flavour mixing and oscillations}

The year 2017 marking the sixteenth anniversary of the possibility of neutrino mixing and oscillations we recall two papers by Bruno Pontecorvo [1,2] where the above mentioned effects have been discussed for the first time. In [1] Pontecorvo has indicated that if the neutrino charge were not conserved then the transition between a neutrino and antineutrino would become possible in vacuum. In [2] Pontecorvo has even directly introduced a phenomenon of neutrino mixing. He has written that

"Neutrinos in vacuum can transform themselves into antineutrino and vice versa. This means that neutrino and antineutrino are particle mixturesïf;. So, for example, a beam of neutral leptons from a reactor which at first consists mainly of antineutrinos will change its composition and at a certain distance $R$ from the reactor will be composed of neutrino and antineutrino in equal quantities".

The paper [2] ends with the following statement

"Under the above assumptions, effects of transformation of neutrino into antineutrino and vice versa may be unobservable in the laboratory because of large value of $R$, but will certainly occur, at least, on an astronomic scale".

A brief history of neutrino mixing and oscillations can be found in [3]. In 1962, just after the discovery of the second flavour neutrino, the effect of neutrino mixing was discussed in [4] where the fields of the weak neutrinos $v_{e}$ and $v_{\mu}$ were connected with the neutrinos mass states $v_{1}$ and $v_{2}$ by the unitary mixing matrix $U$ that can be parametrized by the mixing angle $\theta$ and

$$
v_{e}=v_{1} \cos \theta+v_{2} \sin \theta, \quad v_{\mu}=-v_{1} \sin \theta+v_{2} \cos \theta
$$

The theory of neutrino mixing and oscillations was further developed in $[5,6]$ with actual calculations of neutrino beam evolution. In [7] the effect of neutrino interaction with matter of a constant density on neutrino flavour mixing and oscillations was investigated. The existence of resonant amplification of neutrino mixing (the MSW effect) when a neutrino flux propagates through a medium with varying density was predicted in [8].

The tedious studies, both expertimental and theoretical, over the last 60 years has been honored by the Nobel Prize of 2015 awarded to Arthur McDonald and Takaaki Kajita for the discovery of neutrino oscillations, which shows that neutrinos have mass.

\section{Neutrino spin oscillations}

The straightforward consequences of neutrino nonzero mass is the prediction [9] that neutrinos can have nonzero magnetic moments. Studies of neutrino magnetic moments and the related phenomena attract a reasonable interest in literature. The values of neutrino magnetic moments are constrained in the terrestrial laboratory experiments and in the astrophysical considerations (see, for instance, [10] and [11]).

Massive neutrinos participate in electromagnetic interactions. The recent review on this topic is given in [12]. One of the most important phenomenon of nontrivial neutrino electromagnetic interactions is the neutrino magnetic moment precession and the corresponding spin oscillations in 
presence of external electromagnetic fields. The later effect has been studied in numerous papers published during several passed decades.

Within this scope the neutrino spin oscillations $v^{L} \Leftrightarrow v^{R}$ induced by the neutrino magnetic moment interaction with the transversal magnetic field $\mathbf{B}_{\perp}$ was first considered in [13]. Then spinflavor oscillations $v_{e}^{L} \Leftrightarrow v_{\mu}^{R}$ in $\mathbf{B}_{\perp}$ in vacuum were discussed in [14], the importance of the matter effect was emphasized in [15]. The effect of the resonant amplification of neutrino spin oscillations in $\mathbf{B}_{\perp}$ in the presence of matter was proposed in $[16,17]$, the impact of the longitudinal magnetic field $\mathbf{B}_{\|}$was discussed in [18]. The neutrino spin oscillations in the presence of constant twisting magnetic field were considered in [19-24].

Recently a new approach to description of neutrino spin and spin-flavor oscillations in the presence of an arbitrary constant magnetic field have been developed [24-26]. Within the new approach exact quantum stationary states in the magnetic field are used for classification of neutrino spin states, rather than the neutrino helicity states that have been used for this purpose within the customary approach in many published papers. Recall that the helicity states are not stationary in the presence of a magnetic field. It has been shown [26], in particular, that in the presence of the transversal magnetic field for a given choice of parameters (the energy and magnetic moments of neutrinos and strength of the magnetic field) the amplitude of the flavour oscillations $v_{e}^{L} \leftrightarrow v_{\mu}^{L}$ at the vacuum frequency is modulated by the magnetic field frequency. Similar results on the important influence of the transversal magnetic field on amplitudes of various types of neutrino oscillations were obtained earlier [27] on the basis of the exact solution of the effective equation for neutrino evolution in the presence of a magnetic field and matter, which accounts for four neutrino species corresponding to two different flavor states with positive and negative helicities.

In [28] neutrino spin oscillations were considered in the presence of arbitrary constant electromagnetic fields $F_{\mu \nu}$. Neutrino spin oscillations in the presence of the field of circular and linearly polarized electromagnetic waves and superposition of an electromagnetic wave and constant magnetic field were considered in [29-31].

More general case of neutrino spin evolution in the case when neutrino is subjected to general types of non-derative interactions with external scalar $s$, pseoudoscalar $\pi$, vector $V_{\mu}$, axial-vector $A_{\mu}$, tensor $T_{\mu \nu}$ and pseudotensor $\Pi_{\mu \nu}$ fields was considered in [32]. From the general neutrino spin evolution equation, obtained in [32], it follows that neither scalar $s$ nor pseudoscalar $\pi$ nor vector $V_{\mu}$ fields can induce neutrino spin evolution. On the contrary, within the general consideration of neutrino spin evolution it was shown that electromagnetic (tensor) and weak (axial-vector) interactions can contribute to the neutrino spin evolution.

Recently we consider in details $[25,33]$ neutrino mixing and oscillations in arbitrary constant magnetic field that have $\mathbf{B}_{\perp}$ and $\mathbf{B}_{\|}$nonzero components in mass and flavour bases and derived an explicit expressions for the effective neutrino magnetic moments for the flavour neutrinos in terms of the corresponding magnetic moments introduced in the neutrino mass basis.

\section{Neutrino spin conversion and oscillations in transversal matter current}

For many years, until 2004, it was believed that a neutrino helicity precession and the corresponding spin oscillations can be induced by the neutrino magnetic interactions with an external electromagnetic field that provided the existence of the transversal magnetic field component $\mathbf{B}_{\perp}$ in 
the particles rest frame. A new and very interesting possibility for neutrino spin (and spin-flavour) oscillations engendered by the neutrino interaction with matter background was proposed and investigated for first time in [34]. It was shown [34] that neutrino spin oscillations can be induced not only by the neutrino interaction with a magnetic field, as it was believed before, but also by neutrino interactions with matter in the case when there is a transversal matter current or matter polarization. This new effect has been explicitly highlighted in [34] :

"The possible emergence of neutrino spin oscillations owing to neutrino interaction with matter under the condition that there exists a nonzero transverse current component or matter polarization is the most important new effect that follows from the investigation of neutrino-spin oscillations in Section 4. So far, it has been assumed that neutrino-spin oscillations may arise only in the case where there exists a nonzero transverse magnetic field in the neutrino rest frame."

For historical notes reviewing studies of the discussed effect see in [35,36]. It should be noted that the predicted effect exist regardless of a source of the background matter transversal current or polarization (that can be a background magnetic field, for instance).

Note that the existence of the discussed effect of neutrino spin oscillations engendered by the transversal matter current and matter polarization and its possible impact in astrophysics have been confirmed in a series of recent papers [37-40].

\section{Neutrino spin precession and oscillations in transversal matter currents: semiclassical treatment}

Following the discussion in [34] consider, as an example, an electron neutrino spin precession in the case when neutrinos with the Standard Model interaction are propagating through moving and polarized matter composed of electrons (electron gas) in the presence of an electromagnetic field given by the electromagnetic-field tensor $F_{\mu \nu}=(\mathbf{E}, \mathbf{B})$. To derive the neutrino spin oscillation probability in the transversal matter current we use the generalized Bargmann-Michel-Telegdi equation that describes the evolution of the three-dimensional neutrino spin vector $\mathbf{S}$,

$$
\frac{d \mathbf{S}}{d t}=\frac{2}{\gamma}\left[\boldsymbol{S} \times\left(\boldsymbol{B}_{0}+\boldsymbol{M}_{0}\right)\right],
$$

where the magnetic field $\mathbf{B}_{\mathbf{0}}$ in the neutrino rest frame is determined by the transversal and longitudinal (with respect to the neutrino motion) magnetic and electric field components in the laboratory frame,

$$
\mathbf{B}_{0}=\gamma\left(\mathbf{B}_{\perp}+\frac{1}{\gamma} \mathbf{B}_{\|}+\sqrt{1-\gamma^{-2}}\left[\mathbf{E}_{\perp} \times \frac{\boldsymbol{\beta}}{\beta}\right]\right),
$$

$\gamma=\left(1-\beta^{2}\right)^{-\frac{1}{2}}, \boldsymbol{\beta}$ is the neutrino velocity. The matter term $\mathbf{M}_{0}$ in Eq. (4. (ل) is also composed of the transversal $\mathbf{M}_{0_{\|}}$and longitudinal $\mathbf{M}_{0_{\perp}}$ parts,

$$
\begin{gathered}
\mathbf{M}_{0}=\mathbf{M}_{\mathbf{0}_{\|}}+\mathbf{M}_{\mathbf{0}_{\perp}} \\
\mathbf{M}_{0_{\|}}=\gamma \boldsymbol{\beta} \frac{n_{0}}{\sqrt{1-v_{e}^{2}}}\left\{\rho_{e}^{(1)}\left(1-\frac{\mathbf{v}_{e} \boldsymbol{\beta}}{1-\gamma^{-2}}\right)\right\}-\frac{\rho_{e}^{(2)}}{1-\gamma^{-2}}\left\{\zeta_{e} \boldsymbol{\beta} \sqrt{1-v_{e}^{2}}+\left(\zeta_{e} \mathbf{v}_{e} \frac{\left(\boldsymbol{\beta} \mathbf{v}_{e}\right)}{1+\sqrt{1-v_{e}^{2}}}\right)\right\},
\end{gathered}
$$




$$
\mathbf{M}_{0_{\perp}}=-\frac{n_{0}}{\sqrt{1-v_{e}^{2}}}\left\{\mathbf{v}_{e_{\perp}}\left(\rho_{e}^{(1)}+\rho_{e}^{(2)} \frac{\left(\boldsymbol{\zeta}_{e} \mathbf{v}_{e}\right)}{1+\sqrt{1-v_{e}^{2}}}\right)+\zeta_{e_{\perp}} \rho_{e}^{(2)} \sqrt{1-v_{e}^{2}}\right\} .
$$

Here $n_{0}=n_{e} \sqrt{1-v_{e}^{2}}$ is the invariant number density of matter given in the reference frame for which the total speed of matter is zero. The vectors $\mathbf{v}_{e}$, and $\zeta_{e}\left(0 \leq\left|\zeta_{e}\right|^{2} \leq 1\right)$ denote, respectively, the speed of the reference frame in which the mean momentum of matter (electrons) is zero, and the mean value of the polarization vector of the background electrons in the above mentioned reference frame. The coefficients $\rho_{e}^{(1,2)}$ calculated within the extended Standard Model supplied with $S U(2)$-singlet right-handed neutrino $v_{R}$ are respectively, $\rho_{e}^{(1)}=\frac{\tilde{G}_{F}}{2 \sqrt{2} \mu}, \rho_{e}^{(2)}=-\frac{G_{F}}{2 \sqrt{2} \mu}$, where $\tilde{G}_{F}=G_{F}\left(1+4 \sin ^{2} \theta_{W}\right)$.

For neutrino evolution between two neutrino states $v_{e}^{L} \Leftrightarrow v_{e}^{R}$ in presence of the magnetic field and moving matter we get [34] the following equation

$$
i \frac{d}{d t}\left(\begin{array}{c}
v_{e}^{L} \\
v_{e}^{R}
\end{array}\right)=\mu\left(\begin{array}{cc}
\frac{1}{\gamma}\left|\mathbf{M}_{0 \|}+\mathbf{B}_{0 \|}\right| & \left|\mathbf{B}_{\perp}+\frac{1}{\gamma} \mathbf{M}_{0 \perp}\right| \\
\left|\mathbf{B}_{\perp}+\frac{1}{\gamma} \mathbf{M}_{0 \perp}\right| & -\frac{1}{\gamma}\left|\mathbf{M}_{0 \|}+\mathbf{B}_{0 \|}\right|
\end{array}\right)\left(\begin{array}{c}
v_{e}^{L} \\
v_{e}^{R}
\end{array}\right) .
$$

Thus, the probability of the neutrino spin oscillations in the adiabatic approximation is given by [34]

$$
P_{v_{e}^{L} \rightarrow v_{e}^{R}}(x)=\sin ^{2} 2 \theta_{\mathrm{eff}} \sin ^{2} \frac{\pi x}{L_{\mathrm{eff}}}, \sin ^{2} 2 \theta_{\mathrm{eff}}=\frac{E_{\mathrm{eff}}^{2}}{E_{\mathrm{eff}}^{2}+\Delta_{\mathrm{eff}}^{2}}, \quad L_{\mathrm{eff}}=\frac{2 \pi}{\sqrt{E_{\mathrm{eff}}^{2}+\Delta_{\mathrm{eff}}^{2}}},
$$

where

$$
E_{\text {eff }}=\mu\left|\mathbf{B}_{\perp}+\frac{1}{\gamma} \mathbf{M}_{0 \perp}\right|, \Delta_{\text {eff }}=\frac{\mu}{\gamma}\left|\mathbf{M}_{0 \|}+\mathbf{B}_{0 \|}\right| .
$$

Thus, it follows that even without presence of an electromagnetic field, $\mathbf{B}_{\perp}=\mathbf{B}_{0 \|}=0$, neutrino spin oscillations $v_{e}^{L} \Leftrightarrow v_{e}^{R}$ can be induced in the presence of matter when the transverse matter term $\mathbf{M}_{0 \perp}$ is not zero. If we neglect possible effects of matter polarization then the neutrino evolution equation (4.6) simplifies to

$$
i \frac{d}{d t}\left(\begin{array}{c}
v_{e}^{L} \\
v_{e}^{R}
\end{array}\right)=\frac{\mu}{\gamma}\left(\begin{array}{cc}
M_{0 \|} & M_{0 \perp} \\
M_{0 \perp} & -M_{0 \|}
\end{array}\right)\left(\begin{array}{c}
v_{e}^{L} \\
v_{e}^{R}
\end{array}\right)
$$

where

$$
\mathbf{M}_{0_{\|}}=\gamma \boldsymbol{\beta} \rho_{e}^{(1)}\left(1-\frac{\mathbf{v}_{e} \boldsymbol{\beta}}{1-\gamma^{-2}}\right) \frac{n_{0}}{\sqrt{1-v_{e}^{2}}}, \quad \mathbf{M}_{0_{\perp}}=-\rho_{e}^{(1)} \mathbf{v}_{e_{\perp}} \frac{n_{0}}{\sqrt{1-v_{e}^{2}}} .
$$

The effective mixing angle and oscillation length in the neutrino spin oscillation probability (4.7) now are given by

$$
\sin ^{2} 2 \theta_{\mathrm{eff}}=\frac{M_{0 \perp}^{2}}{M_{0 \|}^{2}+M_{0 \perp}^{2}}, \quad L_{\mathrm{eff}}=\frac{2 \pi}{\mu M_{0}} \gamma
$$

The above considerations can be applied to other types of neutrinos and various matter compositions. It is also obvious that for neutrinos with nonzero transition magnetic moments a similar effect for spin-flavour oscillations exists under the same background conditions. 


\section{Neutrino spin precession and oscillations in transversal matter currents within quantum treatment}

Here below we continue our studies of the effect of neutrino spin evolution induced by the transversal matter currents and develop a consistent derivation of the effect based on the direct calculation of the spin evolution effective Hamiltonian in the case when a neutrino is propagating in matter transversal currents.

Consider two flavour neutrinos with two possible helicities $v_{f}=\left(v_{e}^{+}, v_{e}^{-}, v_{\mu}^{+}, v_{\mu}^{-}\right)^{T}$ in moving matter composed of neutrons. The neutrino interaction Lagrangian reads

$$
L_{i n t}=-f^{\mu} \sum_{l} \bar{v}_{l}(x) \gamma_{\mu} \frac{1+\gamma_{5}}{2} v_{l}(x)=-f^{\mu} \sum_{i} \bar{v}_{i}(x) \gamma_{\mu} \frac{1+\gamma_{5}}{2} v_{i}(x), \quad f^{\mu}=-\frac{G_{F}}{\sqrt{2}} n(1, \mathbf{v}),
$$

where $l=e$, or $\mu, i=1,2$ indicates the neutrino flavour, $i=1,2$ indicates the neutrino mass state and the matter potential $f^{\mu}$ depends on the velocity of matter $\mathbf{v}=\left(v_{1}, v_{2}, v_{3}\right)$ and on the neutron number density in the laboratory reference frame $n=\frac{n_{0}}{\sqrt{1-v^{2}}}$. Each of the flavour neutrinos is a superposition of the neutrino mass states,

$$
v_{e}^{ \pm}=v_{1}^{ \pm} \cos \theta+v_{2}^{ \pm} \sin \theta, \quad v_{\mu}^{ \pm}=-v_{1}^{ \pm} \sin \theta+v_{2}^{ \pm} \cos \theta .
$$

The neutrino evolution equation in the flavour basis is

$$
i \frac{d}{d t} v_{f}=\left(H_{0}^{e f f}+\Delta H^{e f f}\right) v_{f}
$$

where the first term $H_{0}^{\text {eff }}$ of effective Hamiltonian determines the neutrino evolution in nonmoving matter. The second term $\Delta H^{\text {eff }}$ accounts for the effect of matter motion and it can be expressed as

$$
\Delta H^{e f f}=\left(\begin{array}{cccc}
\Delta_{e e}^{++} & \Delta_{e e}^{+-} & \Delta_{e \mu}^{++} & \Delta_{e \mu}^{+-} \\
\Delta_{e e}^{-+} & \Delta_{e e}^{-} & \Delta_{e \mu}^{-+} & \Delta_{e \mu}^{--} \\
\Delta_{\mu e}^{++} & \Delta_{\mu e}^{+-} & \Delta_{\mu \mu}^{++} & \Delta_{\mu \mu}^{+-} \\
\Delta_{\mu e}^{-+} & \Delta_{\mu e}^{--} & \Delta_{\mu \mu}^{-+} & \Delta_{\mu \mu}^{--}
\end{array}\right),
$$

where

$$
\Delta_{k l}^{s s^{\prime}}=\left\langle v_{k}^{s}\left|\Delta H^{S M}\right| v_{l}^{s^{\prime}}\right\rangle, \quad k, l=e, \mu, \quad s, s^{\prime}= \pm .
$$

Note that in the Standard Model interaction Lagrangian (5.D) there is no lepton number violating terms. Therefore, in the Standard Model $\Delta_{k l}^{s s^{\prime}}=0$ for $k \neq l$. Allowing for Non-Standard Interaction of neutrinos [] can generate violation of the lepton flavour universality and as a consequence nonzero terms $\Delta_{k l}^{s s^{\prime}}$ for $k \neq l$ can appear in (5.4).

From (5.]) it follows that

$$
\Delta H^{S M}=\frac{G_{F}}{2 \sqrt{2}} n\left(1+\gamma_{5}\right) \mathbf{v} \boldsymbol{\gamma}, \quad \mathbf{v} \boldsymbol{\gamma}=v_{1} \gamma_{1}+v_{2} \gamma_{2}+v_{3} \gamma_{3}
$$

In evaluation of $\Delta_{k l}^{s s^{\prime}}$ we have first introduced the neutrino flavour states $v_{k}^{s}$ and $v_{l}^{s^{\prime}}$ as superpositions of the mass states $v_{1,2}^{ \pm}$. Then, using the exact free neutrino mass states spinors,

$$
v_{\alpha}^{s}=C_{\alpha} \sqrt{\frac{E_{\alpha}+m_{\alpha}}{2 E_{\alpha}}}\left(\begin{array}{c}
u_{\alpha}^{s} \\
\frac{\sigma p_{\alpha}}{E_{\alpha}+m_{\alpha}} u_{\alpha}^{s}
\end{array}\right) e^{i \boldsymbol{p}_{\alpha} \boldsymbol{x}}, \quad \alpha=1,2,
$$


where the two-component spinors $u_{\alpha}^{s}$

$$
u_{\alpha}^{s=1}=\left(\begin{array}{l}
1 \\
0
\end{array}\right), \quad u_{\alpha}^{s=-1}=\left(\begin{array}{l}
0 \\
1
\end{array}\right)
$$

define neutrino helicity states, we have performed calculations that are analogous to those performed in [33]. The difference in calculations is that here we consider not electromagnetic neutrino interaction with a magnetic field but the neutrino weak interaction with moving matter given by (5.4). For the typical term $\Delta_{\alpha \alpha^{\prime}}^{s s^{\prime}}=\left\langle v_{\alpha}^{s}\left|\Delta H^{S M}\right| v_{\alpha^{\prime}}^{s^{\prime}}\right\rangle$, that by fixing proper values of $\alpha, s, \alpha^{\prime}$ and $s^{\prime}$ can reproduces all of the elements of the neutrino evolution Hamiltonian $\Delta H^{\text {eff }}$ that accounts for the effect of matter motion, we obtain,

$$
\Delta_{\alpha \alpha^{\prime}}^{s s^{\prime}}=\frac{G_{F}}{2 \sqrt{2}} \frac{n_{0}}{\sqrt{1-v^{2}}}\left\{u_{\alpha}^{s T}\left[\left(1-\sigma_{3}\right) v_{\|}+\left(\gamma_{\alpha \alpha^{\prime}}{ }^{-1} \sigma_{1}+i \widetilde{\gamma}_{\alpha \alpha^{\prime}}^{-1} \sigma_{2}\right) v_{\perp}\right] u_{\alpha^{\prime}}^{s^{\prime}}\right\} \delta_{\alpha}^{\alpha^{\prime}},
$$

where $v_{\|}$and $v_{\perp}$ are the longitudinal and transversal velocities of the matter current and

$$
\gamma_{\alpha \alpha^{\prime}}-1=\frac{1}{2}\left(\gamma_{\alpha}^{-1}+\gamma_{\alpha^{\prime}}^{-1}\right), \quad \widetilde{\gamma}_{\alpha \alpha^{\prime}}^{-1}=\frac{1}{2}\left(\gamma_{\alpha}^{-1}-\gamma_{\alpha^{\prime}}^{-1}\right), \quad \gamma_{\alpha}^{-1}=\frac{m_{\alpha}}{E_{\alpha}} .
$$

Recalling expressions for the Pauli matrixes,

$$
\sigma_{3}=\left(\begin{array}{cc}
1 & 0 \\
0 & -1
\end{array}\right), \quad \sigma_{1}=\left(\begin{array}{ll}
0 & 1 \\
1 & 0
\end{array}\right), \quad \sigma_{2}=i\left(\begin{array}{cc}
0 & -1 \\
1 & 0
\end{array}\right),
$$

we get

$$
\Delta_{\alpha \alpha^{\prime}}^{s s^{\prime}}=\frac{G_{F}}{2 \sqrt{2}} \frac{n_{0}}{\sqrt{1-v^{2}}}\left\{u_{\alpha}^{s T}\left[\left(\begin{array}{ll}
0 & 0 \\
0 & 2
\end{array}\right) v_{\|}+\left(\begin{array}{cc}
0 & \gamma_{\alpha}^{-1} \\
\gamma_{\alpha^{\prime}}^{-1} & 0
\end{array}\right) v_{\perp}\right] u_{\alpha^{\prime}}^{s^{\prime}}\right\} \delta_{\alpha}^{\alpha^{\prime}},
$$

The obtained general expression (5.12) can be used for investigations of various types of neutrino spin oscillations in the transversal matter currents considered in the neutrino mass basis. It confirms our prediction [34] that there is the effect of the neutrino spin conversion and corresponding spin oscillations engendered by the interaction with the transversal current of matter. It is also clear that the corresponding effect engendered by the transversal polarization of matter can be treated in much the same way.

On the basis of equation (15.2) and using the relation (5.2) between neutrino mass $v_{\alpha}^{ \pm}$and flavour $v_{l}^{ \pm}$states it is possible to bring our considerations to observational terms and study neutrino oscillations in the flavour basis $v_{f}^{s}$. The neutrino flavour and mass states are connected by the neutrino mixing matrix

$$
v_{f}=U v_{\alpha}
$$

for which we obtain

$$
U=\left(\begin{array}{cccc}
\cos \theta & 0 & \sin \theta & 0 \\
0 & \cos \theta & 0 & \sin \theta \\
-\sin \theta & 0 & \cos \theta & 0 \\
0 & -\sin \theta & 0 & \cos \theta
\end{array}\right)
$$

The corresponding neutrino evolution equation is

$$
i \frac{d}{d t} v_{f}=H_{v}^{f} v_{f}
$$


where the effective Hamiltonian is given by $H_{v}^{f}=U H U^{\dagger}$ and can be directly calculated [42] using equation (5.14).

However, it is possible to get a general structure of the effective evolution Hamiltonian for the flavour neutrino using results of our previous studies [25,33] of neutrino oscillations in arbitrary magnetic field $\boldsymbol{B}=\boldsymbol{B}_{\|}+\boldsymbol{B}_{\perp}$. For evaluation of the flavour neutrino oscillation in an arbitrary moving matter that is characterized by the current $\boldsymbol{j}=\boldsymbol{j}_{\|}+\boldsymbol{j}_{\perp}$ we consider results for the flavour neutrino oscillations in the magnetic field $\boldsymbol{B}$ and account for similarity of the correspondence between the neutrino magnetic moment to magnetic field interaction Hamiltonian $H_{B}$

$$
H_{B}=-\mu_{\alpha \alpha^{\prime}} \bar{v}_{\alpha^{\prime}} \mathbf{\mathbf { B }} v_{\alpha}+\text { h.c. }, \quad \Sigma_{i}=\left(\begin{array}{cc}
\sigma_{i} & 0 \\
0 & \sigma_{i}
\end{array}\right),
$$

and the neutrino to moving matter interaction Hamiltonian $H_{v}$

$$
H_{v}=\widetilde{G} n \bar{v}_{\alpha^{\prime}} \boldsymbol{v} \boldsymbol{\gamma} v_{\alpha}, \quad \widetilde{G}=\frac{G_{F}}{2 \sqrt{2}},
$$

and $n=\frac{n_{0}}{\sqrt{1-v^{2}}}=n_{0} \gamma_{n}$, where $n_{0}$ is the invariant matter density. For the flavour neutrino evolution Hamiltonian in the magnetic field $H_{B}^{f}=U H_{B} U^{\dagger}$ we have ${ }^{1}[25,33]$

$$
H_{B}^{f}=\left(\begin{array}{cccc}
-\left(\frac{\mu}{\gamma}\right)_{e e} B_{\|} & \mu_{e e} B_{\perp} & -\left(\frac{\mu}{\gamma}\right)_{e \mu} B_{\|} & \mu_{e \mu} B_{\perp} \\
\mu_{e e} B_{\perp} & \left(\frac{\mu}{\gamma}\right)_{e e} B_{\|} & \mu_{e \mu} B_{\perp} & \left(\frac{\mu}{\gamma}\right)_{e \mu} B_{\|} \\
-\left(\frac{\mu}{\gamma}\right)_{e \mu} B_{\|} & \mu_{e \mu} B_{\perp} & -\left(\frac{\mu}{\gamma}\right)_{\mu \mu} B_{\|} & \mu_{\mu \mu} B_{\perp} \\
\mu_{e \mu} B_{\perp} & \left(\frac{\mu}{\gamma}\right)_{e \mu} B_{\|} & \mu_{\mu \mu} B_{\perp} & \left(\frac{\mu}{\gamma}\right)_{\mu \mu} B_{\|}
\end{array}\right),
$$

where the following notations are used

$$
\begin{aligned}
\left(\frac{\mu}{\gamma}\right)_{e e} & =\frac{\mu_{11}}{\gamma_{11}} \cos ^{2} \theta+\frac{\mu_{22}}{\gamma_{22}} \sin ^{2} \theta+\frac{\mu_{12}}{\gamma_{12}} \sin 2 \theta, \\
\left(\frac{\mu}{\gamma}\right)_{e \mu} & =\frac{\mu_{12}}{\gamma_{12}} \cos 2 \theta+\frac{1}{2}\left(\frac{\mu_{22}}{\gamma_{22}}-\frac{\mu_{11}}{\gamma_{11}}\right) \sin 2 \theta \\
\left(\frac{\mu}{\gamma}\right)_{\mu \mu} & =\frac{\mu_{11}}{\gamma_{11}} \sin ^{2} \theta+\frac{\mu_{22}}{\gamma_{22}} \cos ^{2} \theta-\frac{\mu_{12}}{\gamma_{12}} \sin 2 \theta \\
\mu_{e e} & =\mu_{11} \cos ^{2} \theta+\mu_{22} \sin ^{2} \theta+\mu_{12} \sin 2 \theta \\
\mu_{e \mu} & =\mu_{12} \cos 2 \theta+\frac{1}{2}\left(\mu_{22}-\mu_{11}\right) \sin 2 \theta \\
\mu_{\mu \mu} & =\mu_{11} \sin ^{2} \theta+\mu_{22} \cos ^{2} \theta-\mu_{12} \sin 2 \theta .
\end{aligned}
$$

The flavour neutrino evolution Hamiltonian in moving matter $H_{v}^{f}=U H_{v} U^{\dagger}$ can be obtained taking into account the mentioned above correspondence of $H_{B}$ and $H_{v}$.

From the above consideration it follows that neutrino spin oscillations can be engendered by the magnetic moment interations with the transversal magnetic field $\boldsymbol{B}_{\perp}$ as well as neutrino weak interactions with the transversal matter current $\boldsymbol{j}_{\perp}$. Consider the initial neutrino state $v_{e}^{L}$ moving

\footnotetext{
${ }^{1}$ The typos in (5.5) and the last formulas of $(5.5)$ and $(\sqrt[5.20]{15})$ as they appear in $[25,33]$ are corrected here.
} 
in the background with the magnetic field $\boldsymbol{B}=\boldsymbol{B}_{\|}+\boldsymbol{B}_{\perp}$ and nonzero matter current $\boldsymbol{j}=\boldsymbol{j}_{\|}+\boldsymbol{j}_{\perp}$. One of the possible modes of neutrino transitions with the change of helicity is $v_{e}^{L} \rightarrow v_{e}^{R}$. The corresponding oscillations are governed by the evolution equation

$$
i \frac{d}{d t}\left(\begin{array}{c}
v_{e}^{L} \\
v_{e}^{R}
\end{array}\right)=\left(\begin{array}{cc}
\left(\frac{\mu}{\gamma}\right)_{e e} B_{\|}+\eta_{e e} \widetilde{G} n(1-v \boldsymbol{\beta}) & \mu_{e e} B_{\perp}+\left(\frac{\eta}{\gamma}\right)_{e e} \widetilde{G} n v_{\perp} \\
\mu_{e e} B_{\perp}+\left(\frac{\eta}{\gamma}\right)_{e e} \widetilde{G} n v_{\perp} & -\left(\frac{\mu}{\gamma}\right)_{e e} B_{\|}-\eta_{e e} \widetilde{G} n(1-\boldsymbol{v} \boldsymbol{\beta})
\end{array}\right)\left(\begin{array}{c}
v_{e}^{L} \\
v_{e}^{R}
\end{array}\right)
$$

where $\left(\frac{\eta}{\gamma}\right)_{e e}$ and $\eta_{e e}$ are given by

$$
\begin{gathered}
\left(\frac{\eta}{\gamma}\right)_{e e}=\left(\frac{\mu}{\gamma}\right)_{e e \mu_{\mu_{11}=\mu_{22}=1, \mu_{12}=0}}=\frac{\cos ^{2} \theta}{\gamma_{11}}+\frac{\sin ^{2} \theta}{\gamma_{22}}, \\
\eta_{e e}=\mu_{e e \mu_{11}=\mu_{22}=1, \mu_{12}=0}=1,
\end{gathered}
$$

if one sets $\mu_{11}=\mu_{22}=1, \mu_{12}=0$ in equations (5.19) and (5.20). For the oscillation $v_{e}^{L} \rightarrow v_{e}^{R}$ probability we get

$$
P_{v_{e}^{L} \rightarrow v_{e}^{R}}(x)=\sin ^{2} 2 \theta_{\mathrm{eff}} \sin ^{2} \frac{\pi x}{L_{\mathrm{eff}}}, \quad \sin ^{2} 2 \theta_{\mathrm{eff}}=\frac{E_{\mathrm{eff}}^{2}}{E_{\mathrm{eff}}^{2}+\Delta_{\mathrm{eff}}^{2}}, \quad L_{\mathrm{eff}}=\frac{2 \pi}{\sqrt{E_{\mathrm{eff}}^{2}+\Delta_{\mathrm{eff}}^{2}}},
$$

where

$$
E_{e f f}=\left|\mu_{e e} \boldsymbol{B}_{\perp}+\left(\frac{\eta}{\gamma}\right)_{e e} \widetilde{G} n \boldsymbol{v}_{\perp}\right|, \Delta_{e f f}=\left|\left(\frac{\mu}{\gamma}\right)_{e e} \boldsymbol{B}_{\|}+\eta_{e e} \widetilde{G} n(1-\boldsymbol{v} \boldsymbol{\beta}) \boldsymbol{\beta}\right| .
$$

In the next section we estimate values of the corresponding parameters that characterize the properties of neutrinos, the background matter and the magnetic field for which neutrino spin oscillations $v_{e}^{L} \Leftrightarrow v_{e}^{R}$, engendered by neutrino weak interactions with the transversal matter current, can proceed with significant probability.

\section{Enhancement of the effect by the longitudinal matter currents and magnetic fields}

We are interested in the situation when the amplitude of oscillations $\sin ^{2} 2 \theta_{\text {eff }}$ in (5.24) is not small and we use the criterion based on the demand that

$$
\sin ^{2} 2 \theta_{\mathrm{eff}}=\frac{E_{\mathrm{eff}}^{2}}{E_{\mathrm{eff}}^{2}+\Delta_{\mathrm{eff}}^{2}} \geq \frac{1}{2},
$$

that is provided by the condition $E_{e f f} \geq \Delta_{e f f}$.

At first we consider the case when the effect of magnetic field is negligible and thus we have

$$
E_{e f f}=\left|\left(\frac{\eta}{\gamma}\right)_{e e} \widetilde{G} n \boldsymbol{v}_{\perp}\right|, \Delta_{e f f}=|\widetilde{G} n(1-\boldsymbol{v} \boldsymbol{\beta}) \boldsymbol{\beta}|
$$

and the oscillation length is given by

$$
L_{e f f}=\frac{2 \pi}{\left(\frac{\eta}{\gamma}\right)_{e e} \widetilde{G} n v_{\perp}}
$$


From the condition $E_{e f f} \geq \Delta_{e f f}$ it follows that

$$
\left(\frac{\eta}{\gamma}\right)_{e e} v_{\perp} \geq(1-\boldsymbol{v} \boldsymbol{\beta})
$$

In the further evaluations we suppose that $\Delta m=m_{2}-m_{1} \ll m_{1}, m_{2}$, and introduce the neutrino effective gamma-factor $\gamma_{v}$

$$
\frac{1}{\gamma_{v}}=\frac{1}{\gamma_{11}} \sim \frac{1}{\gamma_{22}}
$$

Then the condition (6.4) reduces to

$$
\frac{v_{\perp}}{\gamma_{v}} \geq(1-\boldsymbol{v} \boldsymbol{\beta})
$$

Assuming neutrino masses $m_{1}, m_{2} \sim 0.1 \mathrm{eV}$, for a typical neutrino energy $p_{0}^{v} \sim 10 \mathrm{MeV}$ we find $\gamma_{v} \sim 10^{7}$. Consider the case when neutrinos are more relativistic particles than the background matter neutrons $\left(\gamma_{v} \gg \gamma_{n}\right)$, then from (6.6) we get

$$
\frac{1}{\gamma_{v}} \geq \frac{1}{\gamma_{n}^{2}}
$$

The letter condition can be valid for ultra-relativistic background matter with $\gamma_{n} \geq \sqrt{\gamma_{v}} \sim 3 \times 10^{3}$. However, the the oscillation length $L_{\text {eff }}$ given by (6.3) is extremely large.

The presence of the longitudinal magnetic field $\boldsymbol{B}_{\|}$can also have important impact on the criterion (1. I). In the previous consideration the diminishing value of $\Delta_{e f f}$ is attained by the vanishing value of $(1-\boldsymbol{v} \boldsymbol{\beta})$. Now we consider reduction of the term $\widetilde{G} n(1-\boldsymbol{v} \boldsymbol{\beta})$ in $\Delta_{\text {eff }}$ given by (5.25) due to the contribution of $\left(\frac{\mu}{\gamma}\right)_{e e} \boldsymbol{B}_{\|}$. This possibility can be realized when $\boldsymbol{B}_{\|} \boldsymbol{\beta}=-1$. An environment we are considering can be realized by models of short gamma-ray bursts (sGRB) (see [43] and [44]). Consider the neutrino $v_{e}$ escaping the central neutron star with inclination given by an angle $\alpha$ from the plane of the accretion disk. Then this neutrino propagates through the toroidal bulk of very dence matter that rotates with the angular velocity of about $\omega=10^{3} \mathrm{~s}^{-1}$ around the axis that is perpendicular to the accretion disk. The diameter of the perpendicular cut of the toroidal bulk of matter is about $d \sim 20 \mathrm{~km}$ and the distance from the centre of this cut to the centre of the neutron star is also about $D \sim 20 \mathrm{~km}$. The transversal velocity of matter can be estimated accordingly $v_{\perp}=\omega D=0.7$ that corresponds to $\gamma_{n}=1.4$.

Suppose that the direction of the neutrino propagation is characterized by $\sin \alpha \sim \frac{1}{2}$. If there is a magnetic field $B$ perpendicular to the accretion disk then there is the longitudinal field in respect to the neutrino propagation $B_{\|}=B \sin \alpha \sim \frac{1}{2} B$.

In the straightforward analysis we are particular interested in the conversion $v_{e}^{L} \Leftrightarrow v_{e}^{R}$, engendered by interactions with the transversal matter current $\boldsymbol{j}_{\perp}$. Therefore we omit possible effect of the neutrino magnetic moment interaction with the transversal magnetic field $\mu_{e e} \boldsymbol{B}_{\perp}$ in (5.25) and get

$$
E_{e f f}=\left(\frac{\eta}{\gamma}\right)_{e e} \widetilde{G} n v_{\perp}=\widetilde{G} n v_{\perp}\left(\frac{\cos ^{2} \theta}{\gamma_{11}}\right) \approx \widetilde{G} n_{0} \frac{\gamma_{n}}{\gamma_{v}} .
$$

In the considered geometry $\boldsymbol{v} \boldsymbol{\beta}=0$ and for $\Delta_{\text {eff }}$ we use the relation 


$$
\begin{aligned}
\Delta_{e f f} & =\left|\left(\frac{\mu}{\gamma}\right)_{e e} \boldsymbol{B}_{\|}+\eta_{e e} \widetilde{G} n \boldsymbol{\beta}\right|= \\
& =\left|\boldsymbol{B}_{\|}\left(\frac{\mu_{11}}{\gamma_{11}} \cos ^{2} \theta+\frac{\mu_{22}}{\gamma_{22}} \sin ^{2} \theta\right)+\widetilde{G} n \boldsymbol{\beta}\right| \\
& \approx\left|\frac{\mu_{11}}{\gamma_{v}} B_{\|}-\widetilde{G} n_{0} \gamma_{n}\right| .
\end{aligned}
$$

From the demand $E_{e f f}>\Delta_{e f f}$ we get

$$
\left|\frac{\mu_{11} B_{\|}}{\widetilde{G} n_{0} \gamma_{n}}-\gamma_{v}\right|<1
$$

Thus, the criterion (6. I) is fulfilled if the the longitudinal magnetic field is

$$
B_{\|}=B_{\|}^{c r} \sim \gamma_{n} \gamma_{v} \frac{\widetilde{G} n_{0}}{\mu_{11}} .
$$

From the obtained estimation of the critical strength $B_{\|}^{c r}$ of the longitudinal magnetic field $B_{\|}$ follows its important dependence on the matter density $n_{0}$. If one takes an estimation for the neutrino magnetic moment $\mu_{11} \sim 3 \times 10^{-11} \mu_{B}$ and $\gamma_{v}=2 \times 10^{7}$ then in the case of very low matter density $n_{0} \sim 10^{23} \mathrm{~cm}^{-3}$ the critical field is $B_{\|}^{c r} \sim 3 \times 10^{-3} B_{0}$, where $B_{0}=\frac{m_{e}^{2}}{e_{0}}=4.41 \times 10^{13}$ Gauss. For higher densities $n_{0} \sim 10^{32} \mathrm{~cm}^{-3}$ we get $B_{\|}^{c r} \sim 10^{20}$ Gauss. From these estimations it follows that in order to get a reasonable reduction of the term $\widetilde{G} n(1-\boldsymbol{v} \boldsymbol{\beta})$ in $\Delta_{\text {eff }}$ given by (5.25) due to the contribution of $\left(\frac{\mu}{\gamma}\right)_{e e} \boldsymbol{B}_{\| \mid}$the matter density should not be too high to avoid a demand for extremely strong magnetic fields.

Quite recently [46] it has been shown that nonstandard interactions of neutrinos (NIS) with matter [45,47] can significantly alter neutrino flavor evolution in supernovae with the potential to impact explosion dynamics, nucleosynthesis, and the neutrinos signal. Obviously, neutrino spin oscillations can be also engendered by NIS interactions of neutrinos with the transversal matter currents. Different aspects of these very interesting possibilities, including neutrino spin flavour oscillations, are considered elsewhere [48].

\section{Acknowledgments}

The author is thankful to Konstantin Kouzakov, Artem Popov and Pavel Pustoshny for useful discussions. The author is also thankful to George Zoupanos and Margarida Rebelo for the kind invitation to attend the Corfu Summer institute. This work was supported by the Russian Foundation for Basic Research under grants No. 16-02-01023-a and No. 17-52-53133-GFEN.

\section{References}

[1] B. Pontecorvo, Sov. Phys. JETP 6 (1957) 429 [Zh. Eksp. Teor. Fiz. 33 (1957) 549].

[2] B. Pontecorvo, Sov. Phys. JETP 7 (1958) 172 [Zh. Eksp. Teor. Fiz. 34 (1957) 247; JINR Preprint P-45, Dubna, 1957]. 
[3] S. Bilenky, Nucl. Phys. B 908 (2016) 2 doi:10.1016/j.nuclphysb.2016.01.025 [arXiv:1602.00170 [hep-ph]].

[4] Z. Maki, M. Nakagawa and S. Sakata, Prog. Theor. Phys. 28 (1962) 870. doi:10.1143/PTP.28.870

[5] V. N. Gribov and B. Pontecorvo, Phys. Lett. 28B (1969) 493. doi:10.1016/0370-2693(69)90525-5

[6] S. M. Bilenky and B. Pontecorvo, Phys. Lett. 61B (1976) 248. doi:10.1016/0370-2693(76)90141-6

[7] L. Wolfenstein, Phys. Rev. D 17 (1978) 2369. doi:10.1103/PhysRevD.17.2369

[8] S. P. Mikheev and A. Y. Smirnov, Sov. J. Nucl. Phys. 42 (1985) 913 [Yad. Fiz. 42 (1985) 1441].

[9] K. Fujikawa and R. Shrock, Phys. Rev. Lett. 45 (1980) 963.

[10] A. Beda, V. Brudanin, V. Egorov, D. Medvedev et al. Adv. High Energy Phys. 2012 (2012) 350150 .

[11] G. Raffelt, Phys. Rev. Lett. 64 (1990) 2856.

[12] C. Giunti and A. Studenikin, Rev. Mod. Phys. 87 (2015) 531.

[13] A. Cisneros, Astrophys. Space Sci. 10 (1971) 87.

[14] J. Schechter and J. W. F. Valle, Phys. Rev. D 24 (1981) 1883.

[15] L. Okun, M. Voloshin, M. Vysotsky, Sov. J. Nucl. Phys. 44 (1986) 440.

[16] E. Akhmedov, Phys. Lett. B 213 (1988) 64.

[17] C. S. Lim and W. J. Marciano, Phys. Rev. D 37 (1988) 1368.

[18] E. Akhmedov, M. Khlopov, Mod. Phys. Lett. A 3 (1988) 451.

[19] J. Vidal and J. Wudka, Phys. Lett. B 249 (1990) 473. doi:10.1016/0370-2693(90)91019-8

[20] A. Y. Smirnov, Phys. Lett. B 260 (1991) 161. doi:10.1016/0370-2693(91)90985-Y

[21] E. K. Akhmedov, S. T. Petcov and A. Y. Smirnov, Phys. Rev. D 48 (1993) 2167 doi:10.1103/PhysRevD.48.2167 [hep-ph/9301211].

[22] G. G. Likhachev and A. I. Studenikin, J. Exp. Theor. Phys. 81 (1995) 419 [Zh. Eksp. Teor. Fiz. 108 (1995) 769].

[23] M. Dvornikov, J. Phys. G 35 (2008) 025003 doi:10.1088/0954-3899/35/2/025003 [arXiv:0708.2328 [hep-ph]].

[24] A. Dmitriev, R. Fabbricatore and A. Studenikin, PoS CORFU 2014 (2015) 050 [arXiv:1506.05311 [hep-ph]].

[25] A. Studenikin, EPJ Web Conf. 125 (2016) 04018. doi:10.1051/epjconf/201612504018

[26] A. Popov and A. Studenikin, arXiv:1803.05755 [hep-ph].

[27] P. Kurashvili, K. A. Kouzakov, L. Chotorlishvili and A. I. Studenikin, Phys. Rev. D 96 (2017) no.10, 103017 doi:10.1103/PhysRevD.96.103017 [arXiv:1711.04303 [hep-ph]].

[28] A. Egorov, A. Lobanov and A. Studenikin, Phys. Lett. B 491 (2000) 137.

[29] A. Lobanov and A. Studenikin, Phys. Lett. B 515 (2001) 94.

[30] M. S. Dvornikov and A. I. Studenikin, Phys. Atom. Nucl. 64 (2001) 1624 [Yad. Fiz. 64 (2001) 1705]. doi:10.1134/1.1409503 
[31] M. S. Dvornikov and A. I. Studenikin, Phys. Atom. Nucl. 67 (2004) 719 [Yad. Fiz. 67 (2004) 741 ]. doi:10.1134/1.1707133

[32] M. Dvornikov, A. Studenikin, JHEP 09 (2002) 016.

[33] R. Fabbricatore, A. Grigoriev and A. Studenikin, J. Phys. Conf. Ser. 718 (2016) 062058 doi:10.1088/1742-6596/718/6/062058 [arXiv:1604.01245 [hep-ph]].

[34] A. Studenikin, Phys. Atom. Nucl. 67 (2004) 993.

[35] A. Studenikin, arXiv:1610.06563 [hep-ph].

[36] A. Studenikin, J. Phys. Conf. Ser. 718 (2016) 062076 doi:10.1088/1742-6596/718/6/062076 [arXiv:1603.00337 [hep-ph]].

[37] V. Cirigliano, G. Fuller, A. Vlasenko, Phys. Lett. B 747 (2015) 27.

[38] C. Volpe, Int. J. Mod. Phys. E 24 (2015) 1541009.

[39] A. Kartavtsev, G. Raffelt, H. Vogel, Phys. Rev. D 91 (2015) 125020.

[40] A. Dobrynina, A. Kartavtsev, G. Raffelt, Phys. Rev. D 93 (2016) 125030.

[41] A. Studenikin, PoS NOW 2016 (2016) 070 [arXiv:1706.01100 [hep-ph]].

[42] A. Popov, P. Pustoshny, A. Studenikin

[43] A. Grigoriev, A. Lokhov, A. Studenikin and A. Ternov, JCAP 1711 (2017) no.11, 024 doi:10.1088/1475-7516/2017/11/024 [arXiv:1705.07481 [hep-ph]].

[44] A. Perego, S. Rosswog, R. M. Cabezï£;n, O. Korobkin, R. Kï£ippeli, A. Arcones and M. Liebendïjrfer, Mon. Not. Roy. Astron. Soc. 443 (2014) no.4, 3134 doi:10.1093/mnras/stu1352 [arXiv:1405.6730 [astro-ph.HE]].

[45] T. Ohlsson, Rept. Prog. Phys. 76 (2013) 044201 doi:10.1088/0034-4885/76/4/044201 [arXiv:1209.2710 [hep-ph]].

[46] C. J. Stapleford, D. J. Vï£;ï£¡nï£¡nen, J. P. Kneller, G. C. McLaughlin and B. T. Shapiro, Phys. Rev. D 94 (2016) no.9, 093007 doi:10.1103/PhysRevD.94.093007 [arXiv:1605.04903 [hep-ph]].

[47] Y. Farzan and M. Tortola, Front. in Phys. 6 (2018) 10 doi:10.3389/fphy.2018.00010 [arXiv:1710.09360 [hep-ph]].

[48] P. Pustoshny, A. Studenikin, in preparation. 